Research Article

\title{
Geometric Mechanics on Warped Product Semi-Slant Submanifold of Generalized Complex Space Forms
}

\author{
Yanlin Li $\mathbb{D}^{1},{ }^{1}$ Ali H. Alkhaldi, ${ }^{2}$ and Akram Ali $\mathbb{D}^{3}$ \\ ${ }^{1}$ School of Mathematics, Hangzhou Normal University, Hangzhou 311121, China \\ ${ }^{2}$ Department of Mathematics, College of Science, King Khalid University, 9004 Abha, Saudi Arabia \\ ${ }^{3}$ Department of Mathematics, College of Science, King Khalid University, 61413 Abha, Saudi Arabia
}

Correspondence should be addressed to Akram Ali; akramali133@gmail.com

Received 22 August 2021; Accepted 27 September 2021; Published 5 November 2021

Academic Editor: John D. Clayton

Copyright (c) 2021 Yanlin Li et al. This is an open access article distributed under the Creative Commons Attribution License, which permits unrestricted use, distribution, and reproduction in any medium, provided the original work is properly cited.

In this study, we develop a general inequality for warped product semi-slant submanifolds of type $M^{n}=N_{T}^{n_{1}} \times{ }_{f} N_{\vartheta}^{n_{2}}$ in a nearly Kaehler manifold and generalized complex space forms using the Gauss equation instead of the Codazzi equation. There are several applications that can be developed from this. It is also described how to classify warped product semi-slant submanifolds that satisfy the equality cases of inequalities (determined using boundary conditions). Several results for connected, compact warped product semi-slant submanifolds of nearly Kaehler manifolds are obtained, and they are derived in the context of the Hamiltonian, Dirichlet energy function, gradient Ricci curvature, and nonzero eigenvalue of the Laplacian of the warping functions.

\section{Introduction}

We can examine the energy, angles, and lengths of their second fundamental form using certain warped product manifolds. These manifolds are generalizations of Riemannian product manifolds and provide examples of manifolds with a strictly negative curvature from a mathematical standpoint. They can be usefully applied to models of spacetime around black holes and bodies with enormous gravitational fields from a mechanical standpoint. From the geometric standpoint of applied mathematics, their warping functions can solve numerous partial differential equations (see $[1,2])$. Bishop and O'Neill [3] first proposed the concept of warped product manifolds in order to analyze negative curvature manifolds. Here's how they define it.

Definition 1. Let $\left(N_{1}, g_{1}\right)$ and $\left(N_{2}, g_{2}\right)$ be two Riemannian manifolds, where $f: N_{1} \longrightarrow(0, \infty)$ and $\gamma_{1}: N_{1} \times N_{2} \longrightarrow$ $N_{1}, \gamma_{2}: N_{1} \times N_{2} \longrightarrow N_{2}$; the orthogonal projection maps are defined as $\gamma_{1}(t, s)=t$ and $\gamma_{2}(t, s)=s$ for any $(t, s) \in N_{1}$ $\times N_{2}$. Then, the warped product $N_{1} \times{ }_{f} N_{2}$ is a product manifold that is $N_{1} \times N_{2}$ associated with the Riemannian structure; in other words,

$$
g(X, Y)=g_{1}\left(\gamma_{1} * X, \gamma_{1} * Y\right)+\left(f \circ \gamma_{1}\right)^{2} g_{2}\left(\gamma_{2} * X, \gamma_{2} * Y\right),
$$

for any $X, Y \in T M^{n}$, where the tangent map is denoted by * and $f$ represents a warping function of $M^{n}$.

The theory of slant submanifolds is currently under investigation; it was first established by Chen in [4] for nearly Hermitian manifolds. The almost complex (holomorphic) and entirely real submanifolds are specific examples among the classes of slant submanifolds. As a result, the warped product semi-slant submanifold is the most basic generalization of a CR-warped product submanifold. alSolamy et al. [5] recently investigated a warped product semi-slant submanifold of a nearly Kaehler manifold, proving that no such warped product semi-slant submanifold exists of the form $M^{n}=N_{\vartheta}^{n_{2}} \times{ }_{f} N_{T}^{n_{1}}$, where $N_{\vartheta}^{n_{2}}$ is a proper slant submanifold and $N_{T}^{n_{1}}$ is a complex submanifold.

The researchers next looked into warped products of the type $M^{n}=N_{T}^{n_{1}} \times{ }_{f} N_{\vartheta}^{n_{2}}$ and came up with a number of fascinating conclusions, including characterizations and an inequality. We refer to [6] for a survey of warped product submanifolds. We first remark that the utilization of the 
Codazzi equation in Chen's study [7] problem atomizes attempts to extend its results from the warped product semi-slant submanifold setting, due to the slant angle's involvement. We use a novel strategy in this work, substituting the Codazzi equation (used in [7]) with the Gauss equation. As a generalization of the contact CR-warped products, we construct a sharp general inequality for warped product semi-slant submanifolds isometrically immersed in a generalized space form. We also investigate nontrivial warped product semi-slant submanifolds of type $M^{n}=N_{\vartheta}^{n_{2}} \times{ }_{f} N_{T}^{n_{1}}$ that are isometrically immersed in an arbitrary nearly Kaehler manifold; we obtain results (cf. Theorem 21) and consider interesting applications thereof (cf. Theorem 22).

Chen developed a general inequality for the CRwarped product of complex space forms in [7]. Furthermore, in [8-11], the classifications of contact CR-warped products in spheres that satisfy the equality cases similarly were given. The classifications of the totally geodesic and totally umbilical submanifolds are examples of how these relations might be used to classify equalities in the derived inequality. For various types of inequalities, several authors (in [12-17]) have presented thorough classifications of CR-warped products in complex projective space forms and Lagrangian submanifolds in complex space forms. Motivated by previous studies, we derived necessary and sufficient conditions to determine whether a compact oriented warped product semi-slant submanifold in a generalized complex space forms is trivial (cf. Theorems 24, 25, and 26, and Corollaries 27 and 28).

Calin and Chang presented a geometric approach to Riemannian manifolds in [18], identifying its applicability to partial differential equations that implement a Lagrangian formalism on Riemannian manifolds; for example, they considered its application to the energy-momentum tensor and conservation laws; the Hamiltonian formalism; HamiltonJacobi theory; harmonic functions, maps, and geodes; and harmonic functions, maps, and geodes. Let us note that the geometry of a Riemannian manifold can be thought of as a compact Riemannian submanifold with a boundary; in other words, $\partial M \neq \varnothing$. We considered the Euler-Lagrange equation, kinetic energy function, and Hamiltonian approach to warped product submanifolds for which the warping function plays an important role as a positive differential function for such identities because of the influence of the slant angle in a warped product semi-slant submanifold of a nearly Kaehler we provide (cf. Theorems 29, 30, and 32).

The effect of Ricci curvature on the structure of warped products is investigated. In Riemannian geometry, one important question arises: What is the geometric meaning of Ricci curvature? Answer: Ricci-flat manifolds require us to solve the Riemannian manifold's Einstein field equations with a vanishing cosmological constant geometrically.

We study the Ricci curvature on the structure of warped products. One fundamental question arises: What is the geometric meaning of Ricci curvature in Riemannian geometry? Answer: Geometrically, Ricci-flat manifolds require us to solve the Einstein field equations of the Riemannian manifold with a vanishing cosmological constant. In general relativity, the Ricci tensor corresponds to the universe's matter content via Einstein field equations. The degree to which matter tends to converge or diverge over time is determined by this term of spacetime curvature. As a result, in physics, Ricci curvature is more essential than Riemannian curvature, and geometric obstacles of the Ricci curvature and Ricci tensor will be found in warped product manifolds (for further details, see [12,19] and the references therein). Our next goal is to look into the physical implications of these issues in terms of warping functions. We propose our result (cf. Theorem 33) to enable our study to uncover the useful applications of the obtained inequality in physics. The work described in this paper will be combined with the singularity theory techniques presented in [20-24].

The following is a breakdown of the paper's structure: We review some basic formulas and definitions in Section 2 and give a quick overview of semi-slant submanifolds. In Section 3, we analyze warped product semi-slant submanifolds and prove an inequality for an intrinsic invariant in a nearly Kaehler manifold in terms of the second basic form, the squared norm of the warping function, and the Laplacian of the warping functions. The case of equality is also examined. In this section, we get the main result for warped product semi-slant submanifolds immersed isometrically in a nearly Kaehler manifold. In Section 4, we use boundary conditions to explain multiple classifications of such inequalities for Riemannian and compact Riemannian submanifolds. In Section 5, we strengthen the second fundamental form inequality in a virtually Kaehler manifold for warped product semi-slant submanifolds and CR-warped product submanifolds. We also show that the warped product semi-slant manifold in a nearly Kaehler manifold becomes a Riemannian product under a set of complicated requirements expressed in terms of the kinetic energy function and the Hamiltonian of the warping function. In Section 6 , we prove that the compact warped product semi-slant submanifold of a virtually Kaehler manifold is either a CR-warped product manifold or a simple Riemannian product manifold in terms of the gradient Ricci curvature of warped functions.

\section{Preliminaries}

An almost Hermitian manifold $(\tilde{M}, J, g)$ of a $2 m$-dimensional space, such that $J$ is an almost complex structure and $g$ is a Riemannian metric, satisfies

$$
(a) J^{2}=-I,(b) g(J X, J Y)=g(X, Y),
$$

for any $X, Y$ on $\tilde{M}^{2 m}$, where the identity map is denoted by $I$. Let $\Gamma\left(T \tilde{M}^{2 m}\right)$ denote the set of all vector fields tangent to $\tilde{M}^{2 m}$; the Levi-Civita connection defined on $\tilde{M}^{2 m}$ is denoted by $\tilde{\nabla}$. Then, a Kaehler manifold with an almost complex structure $J$ satisfies

$$
\left(\nabla_{X}^{\sim} J\right) Y=0
$$


for any $X, Y \in \Gamma\left(T \tilde{M}^{2 m}\right)$ and $\tilde{M}^{2 m}$. Moreover, if the almost complex structure $J$ is such that

$$
\left(\nabla_{X} J\right) Y+\left(\nabla_{Y} J\right) X=0
$$

for any vector field $X, Y$ tangent to $\tilde{M}^{2 m}$, then the manifold $\tilde{M}$ represents a nearly Kaehler manifold [25-27]. The above equation is similar to the following:

$$
\left(\nabla_{X} J\right) X=0, \quad X \in \Gamma\left(T \tilde{M}^{2 m}\right) .
$$

Assume that $\tilde{M}^{n}$ is a complex space form of constant holomorphic sectional curvature $4 \kappa$ and it is denoted by $\tilde{M}^{n}(4 \kappa)$. The curvature tensor $\tilde{R}$ of $\tilde{M}^{n}(4 \kappa)$ can be expressed as

$$
\begin{aligned}
\tilde{R}\left(W_{1}, W_{2}\right) W_{3}= & \kappa\left\{g\left(W_{2}, W_{3}\right) W_{1}-g\left(W_{1}, W_{3}\right) W_{2}\right. \\
& +g\left(W_{3}, J W_{2}\right) J W_{1}-g\left(W_{3}, J W_{1}\right) J W_{2} \\
& \left.+2 g\left(W_{1}, J W_{2}\right) J W_{3}\right\},
\end{aligned}
$$

for all $W_{1}, W_{2}, W_{3} \in \Gamma(T \tilde{N})$. Based on the cases $\kappa<0, \kappa$ $=0$, and $\kappa>0, \widetilde{\mathbb{N}}^{n}(4 \kappa)$ is the complex hyperbolic space $\mathbb{C}$ $H^{n}$, complex Euclidean space $\mathbb{C}^{n}$, or the complex projective space $\mathbb{C} P^{n}$. Now, we consider the generalized complex space forms which are a natural generality of complex space forms and a special family of Hermitian manifolds. Actually, a generalized complex space form is a $R K$-manifold of constant type $\alpha$ with constant holomorphic sectional curvature $\kappa$. Moreover, it is denoted by $\tilde{M}^{2 m}(\kappa, \alpha)$. Hence, the curvature tensor $\tilde{R}$ for generalized complex space is given by

$$
\begin{aligned}
\tilde{R}(X, Y, Z, W)= & \frac{\kappa+3 \alpha}{4}\{g(Y, Z) g(X, W)-g(Y, W) g(X, Z)\} \\
& +\frac{\kappa-\alpha}{4}\{g(X, J Z) g(J Y, W)-g(Y, J Z) g(J X, W) \\
& +2 g(X, J Y) g(J Z, W)\},
\end{aligned}
$$

for any $X, Y, Z, W \in \Gamma\left(T \tilde{M}^{2 m}\right)$. Thus, for the more classifications of generalized complex space forms, we refer to $[10,11,28-32]$. The curvature tensor $\tilde{R}$ for a nearly Kaehler 6 -sphere is given by

$$
\tilde{R}(X, Y, Z, W)=g(Y, Z) g(X, W)-g(Y, W) g(X, Z),
$$

for any $X, Y, Z, W\left(T \tilde{M}\left(\mathbb{S}^{6}\right)\right.$.

A submanifold is denoted by the $M^{n}$ of an almost Hermitian manifold $\tilde{M}^{2 m}$ with an induced Riemannian metric g. However, $\nabla^{\perp}$ and $\nabla$ represent the induced Riemannian connections on the normal bundle $T^{\perp} M^{n}$ and tangent bundle $T M^{n}$ of $M^{n}$, respectively. Thus, the Gauss and Weingarten formulas are defined as

$$
\text { (i) } \nabla^{\sim}{ }_{X} Y=\nabla_{X} Y+h(X, Y), \quad \text { (ii) } \nabla^{\sim}{ }_{X} N=-A_{N} X+\nabla_{X}^{\perp} N \text {, }
$$

for every $N \in \Gamma\left(T^{\perp} M^{n}\right)$ and $X, Y \in \Gamma\left(T M^{n}\right)$, where $A_{N}$ and $h$ denote the shape operator and second fundamental form for an immersion of $M$ into $\tilde{M}^{2 m}$, respectively. Now, for any $N \in \Gamma\left(T^{\perp} M^{n}\right)$ and $X \in \Gamma\left(T M^{n}\right)$, we have

$$
\text { (i) } J X=P X+F X, \quad \text { (ii) } J N=t N+f N \text {, }
$$

where $F X(f N)$ and $P X(t N)$ are the normal and tangential components of $J N(J X)$, respectively. From (2), it can be clearly seen that, for each $X, Y \in \Gamma\left(T M^{n}\right)$, we have

$$
\text { (a) } g(P X, Y)=-g(X, P Y), \quad(b)\|P\|^{2}=\sum_{i, j=1}^{n} g^{2}\left(P e_{i}, e_{j}\right),
$$

for each $e_{i}, i=1, \cdots, n$ tangent to $M^{n}$. Assuming $\tilde{M}^{2 m}$ to be a Riemannian manifold and $M^{n}$ a submanifold of $\tilde{M}^{2 m}$, the Gauss equation can be defined as

$$
\begin{aligned}
\tilde{R}(X, Y, Z, W)= & R(X, Y, Z, W)+g(h(X, Z), h(Y, W)) \\
& -g(h(X, W), h(Y, Z)),
\end{aligned}
$$

for any $X, Y, Z, W \in \Gamma\left(T M^{n}\right)$, where $\tilde{R}$ and $R$ represent the curvature tensors on $\tilde{M}^{2 m}$ and $M^{n}$, respectively. Furthermore, totally umbilical and totally geodesic submanifolds satisfy $h(X, Y)=g(X, Y) H$ and $h(X, Y)=0$, respectively, for any $X, Y \in \Gamma\left(T M^{n}\right)$, where $H$ is the mean curvature vector of $M^{n}$. If $H=0$, then $M^{n}$ is called a minimal submanifold. The mean curvature vector $H$ is expressed in terms of $\left\{e_{1}\right.$, $\left.e_{2} \cdots, e_{n}\right\}$, which is the so-called orthonormal frame of the tangent space $T M^{n}$; it is defined as

$$
H=\frac{1}{n} \operatorname{trace}(h)=\frac{1}{n} \sum_{i=1}^{n} h\left(e_{i}, e_{i}\right)
$$

where $n=\operatorname{dim} M$. Moreover, we have

$$
\|h\|^{2}=\sum_{i, j=1}^{n} g\left(h\left(e_{i}, e_{j}\right), h\left(e_{i}, e_{j}\right)\right), \quad h_{i j}^{r}=g\left(h\left(e_{i}, e_{j}\right), e_{r}\right),
$$

for which $\left\{e_{i}\right\}_{i=1, \cdots n}$ and $\left\{e_{r}\right\}_{r=n+1, \cdots 2 m}$ are orthonormal frames tangent to $M^{n}$ and normal to $M^{n}$, respectively. The scalar curvature $\tau$ for a submanifold $M^{n}$ of an almost complex manifold $\tilde{M}^{2 m}$ is given by

$$
\tau\left(T M^{n}\right)=\sum_{1 \leq i \neq j \leq n} K\left(e_{i} \wedge e_{j}\right) .
$$


In the above equation, $e_{i}$ and $e_{j}$ represent the span of the plane section, and its sectional curvature is denoted by $K$ $\left(e_{i} \wedge e_{j}\right)$. Let $G_{r}$ be an $r$-plane section on $T M^{n}$ and $\left\{e_{1}\right.$, $\left.e_{2} \cdots e_{r}\right\}$ be any orthonormal basis of $G_{r}$. Then, the scalar curvature $\tau\left(G_{r}\right)$ of $G_{r}$ is defined as

$$
\tau\left(G_{r}\right)=\sum_{1 \leq i \neq j \leq r} K\left(e_{i} \wedge e_{j}\right)
$$

Let $\phi$ be a differential function defined on $M^{n}$. Thus, the gradient $\vec{\nabla} \phi$ is given as

$$
g(\vec{\nabla} \phi, X)=X f, \quad \vec{\nabla} \phi=\sum_{i=1}^{n} e_{i}(\phi) e_{i}
$$

Thus, from the above equation, the Hamiltonian in a local orthonormal frame is defined as

$$
H(\mathrm{~d} \phi, x)=\frac{1}{2} \sum_{j=1}^{n} d f\left(e_{j}\right)^{2}=\frac{1}{2} \sum_{j=1}^{n} e_{j}(\phi)^{2}=\frac{1}{2}\|\nabla \phi\|^{2} .
$$

Moreover, the Laplacian $\Delta f$ of $f$ is also given by

$$
\Delta \phi=\sum_{i=1}^{n}\left\{\left(\nabla_{e_{i}} e_{i}\right) \phi-e_{i}\left(e_{i}(\phi)\right)\right\}=-\sum_{i=1}^{n} g\left(\nabla_{e_{i}} \operatorname{grad} \phi, e_{i}\right) \text {. }
$$

Similarly, the Hessian tensor of function $f$ is given by

$$
\Delta \phi=-\operatorname{Trace} H^{\phi}=-\sum_{i=1}^{n} \operatorname{Hess}(\phi)\left(e_{i}, e_{i}\right),
$$

where $H^{\phi}$ denotes the Hessian tensor. The compact manifold $M^{n}$ is considered as being without a boundary; that is, $\partial M^{n}=\varnothing$. Thus, we have the following lemma.

Lemma 2 (see [18]; Hopf's lemma). Let $M^{n}$ be a connected and compact Riemannian manifold and $\phi$ a smooth function on $M^{n}$ such that $\Delta \phi \geq 0(\Delta \phi \leq 0)$. Then, $\phi$ is a constant function on $M^{n}$.

Moreover, the integration of the Laplacian of the smooth function, defined on a compact-orientated Riemannian manifold $M^{n}$ without boundary, vanishes with respect to the volume element of such a manifold, and we obtain the following formula:

$$
\int_{M^{n}} \Delta \phi \mathrm{d} V=0
$$

where $d V$ denotes the volume of $M^{n}$ (see [33]).

Theorem 3 (see [18]). The Euler-Lagrange equation for the Lagrangian is

$$
\Delta \phi=0
$$

Hopf's lemma becomes the uniqueness theorem for the Dirichlet problem if manifold $M^{n}$ has a boundary.

Theorem 4 (see [18]). Let $M^{n}$ be a connected and compact manifold and $f$ a positive differentiable function on $M^{n}$ such that $\Delta \phi=0, O n M^{n}$. Thus, $\phi=0$, where $\partial M^{n}$ is the boundary of $M$.

Moreover, let $M^{n}$ be a compact Riemannian manifold and $f$ be a positive differentiable function on $M^{n}$. Then, the Dirichlet energy function is defined as described in [18]; that is,

$$
E(\phi)=\frac{1}{2} \int_{M^{n}}\|\nabla \phi\|^{2} \mathrm{~d} V
$$

If $M^{n}$ is compact, then $0 \leq E(\phi)<\infty$. We provide the following definition of a slant submanifold.

Definition 5 (see [4]). Assume $T_{x} M^{n}-\{0\}$ to be a set containing all nonzero tangent vector fields of immersion $M^{n}$ in an almost Hermitian manifold $\tilde{M}^{2 m}$ at a point $x \in M^{n}$. Then, for each vector $X \in\left(T_{x} M^{n}\right)$ at point $x \in M^{n}$, the angle between JX and the tangent space $T_{x} M$ is considered to be the Wirtinger angle of $X$ at $x \in M^{n}$; this is denoted as $\vartheta(X)$. In this case, a submanifold $M^{n}$ of $\tilde{M}^{2 m}$ is called a slant submanifold such that $\vartheta$ is a slant angle.

It is clear that the slant submanifolds include totally real and holomorphic submanifolds. However, Chen proved the following characterization theorem of slant submanifolds.

Theorem 6. Let $\tilde{M}^{2 m}$ be an almost Hermitian manifold and $M^{n}$ be a submanifold of $\tilde{M}^{2 m}$. Then, $M^{n}$ is slant if and only if there exists a constant $\lambda \in[0,1]$ such that

$$
P^{2}=-\lambda I
$$

where $\lambda=\cos ^{2} \vartheta$ for a slant angle $\vartheta$ defined on the tangent bundle $T M^{n}$ of $M^{n}$.

Hence, we have the following consequences of Theorem 6:

$$
\begin{aligned}
& g(P X, P Y)=\cos ^{2} \vartheta g(X, Y), \\
& g(F X, F Y)=\sin ^{2} \vartheta g(X, Y),
\end{aligned}
$$

for any $X, Y \in \Gamma\left(T M^{n}\right)$.

In an essentially Hermitian manifold, another group of submanifolds known as semi-slant submanifolds exists as a natural generalization of slant submanifolds, CR-submanifolds, and holomorphic and antiholomorphic submanifolds. Papaghiuc researched and defined semi-slant submanifolds in [34] as a natural extension of CR-submanifolds of an almost Hermitian manifold. The following is the definition of a semi-slant submanifold. 
Definition 7. A Riemannian submanifold $M^{n}$ of an almost Hermitian manifold $\tilde{M}^{2 m}$ is defined as a semi-slant submanifold if there exist two complementary distributions $\mathscr{D}$ and $\mathscr{D}^{9}$ such that

(i) $T M=\mathscr{D} \oplus \mathscr{D}^{9}$

(ii) $\mathscr{D}$ is a holomorphic distribution; that is, $J(\mathscr{D})=\mathscr{D}$

(iii) $\mathscr{D}^{9}$ is called a slant distribution if slant angle $\vartheta \neq 0, \pi / 2$

Remark 8. For a semi-slant submanifold, let us consider the dimensions of $\mathscr{D}$ and $\mathscr{D}^{9}$ in $d_{1}$ and $d_{2}$; then, $M^{n}$ is holomorphic if $d_{2}=0$ and slant if $d_{1}=0$. Furthermore, if $\vartheta=\pi$ / 2 and $d_{1}=0$, then $M^{n}$ is represented as an antiholomorphic (totally real) submanifold. Moreover, $M^{n}$ is referred to as a proper semi-slant submanifold if $\vartheta$ differs from 0 and $\pi / 2$. We can also define $M^{n}$ as proper if $d_{1} \neq 0$ and $d_{2} \neq 0$.

Remark 9. If $v$ is an invariant normal subspace under an almost complex structure $J$ of the normal bundle $T^{\perp} M^{n}$, then this normal bundle is decomposed as

$$
T^{\perp} M^{n}=F \mathscr{D}^{9} \oplus \nu
$$

\section{Warped Product semi-slant Submanifolds of Nearly Kaehler Manifolds}

We will go through some of the findings on warped product manifolds in this section. References [5, 6, 35-40] provide more information. We derive our main inequality for the squared norm of the second fundamental form in terms of constant holomorphic sectional curvature using numerous geometric conditions for the mean curvature of a warped product semi-slant submanifold.

In particular, a warped product manifold is classified to be trivial if the warping function is constant. In such cases, we refer to the warped product manifold as a Riemannian product manifold. It was proven in [3] that, for $X \in \Gamma\left(T N_{1}\right)$ and $Z \in \Gamma\left(T N_{2}\right)$, the following is satisfied:

$$
\nabla_{X} Z=\nabla_{Z} X=(X \ln f) Z
$$

where $\nabla$ denotes the Levi-Civita connection on $M^{n}$. We recall the following lemma obtained in [3].

Lemma 10. A warped product manifold $M^{n}=N_{1} \times{ }_{f} N_{2}$. Thus,

(i) $\nabla_{X} Y \in \Gamma\left(T N_{1}\right)$

(ii) $\nabla_{Z} W=\nabla_{Z}^{\prime} W-g(Z, W) \nabla \ln f$

for any $Z, W \in \Gamma\left(T N_{2}\right)$ and $X, Y \in \Gamma\left(T N_{1}\right)$, where $\nabla^{\prime}$ is the Levi-Civita connection on $\mathrm{N}_{2}$.
Remark 11. If the warping function $f$ is constant, then $M$ $=N_{1} \times{ }_{f} N_{2}$ is a trivial warped product or a simple Riemannian product.

Remark 12. In a nontrivial warped product manifold $M^{n}=$ $N_{1} \times{ }_{f} N_{2}$, the manifold $N_{1}$ is totally geodesic, and $N_{2}$ is a totally umbilical submanifold in $M^{n}$.

Let $\varphi: M^{n}=N_{1} \times{ }_{f} N_{2} \longrightarrow \tilde{M}^{2 m}$ be an isometric immersion of a warped product manifold $N_{1} \times{ }_{f} N_{2}$ in an arbitrary Riemannian manifold $\tilde{M}^{2 m}$. Furthermore, let $n_{1}, n_{2}$, and $n$ represent the real dimensions of $N_{1}, N_{2}$, and $M^{n}$, respectively. Then, for any unit tangent vectors $Y$ and $W$ on $N_{1}$ and $N_{2}$, respectively, we have

$K(Y \wedge W)=g\left(\nabla_{W} \nabla_{Y} Y-\nabla_{Y} \nabla_{W} Y, W\right)=\frac{1}{f}\left\{\left(\nabla_{Y} Y\right) f-Y^{2} f\right\}$

If we consider the local orthonormal frame $\left\{e_{1}, e_{2}, \cdots, e_{n}\right\}$ such that the vectors $e_{1}, e_{2} \cdots, e_{n_{1}}$ are tangential to $N_{1}$ and $e_{n_{1}+1} \cdots, e_{n}$ are tangential to $N_{2}$, then in view of the Gauss equation (12), we can deduce that

$$
\tau\left(T M^{n}\right)=\tilde{\tau}\left(T M^{n}\right)+\sum_{r=1}^{2 m} \sum_{1 \leq i \neq j \leq n}\left(h_{i i}^{r} h_{j j}^{r}-\left(h_{i j}^{r}\right)^{2}\right)
$$

for each $j=n_{1}+1 \cdots n$.

Hereafter, we will denote the corresponding dimensions as indices. Recall that [5] proved several results for both types of warped product semi-slant submanifolds in nearly Kaehler manifolds.

Theorem 13 (see [5]). There does not exist a proper warped product submanifold of the form $M^{n}=N_{\vartheta}^{n_{2}} \times{ }_{f} N_{T}^{n_{1}}$ in a nearly Kaehler manifold $\tilde{M}^{2 m}$ such that $N_{\vartheta}^{n_{2}}$ is a proper slant submanifold and $N_{T}^{n_{1}}$ is a holomorphic submanifold of $\tilde{M}^{2 m}$.

Lemma 14. For a nontrivial warped product semi-slant submanifold $M^{n}=N_{T}^{n_{1}} \times{ }_{f} N_{\vartheta}^{n_{2}}$ in a nearly Kaehler manifold $\tilde{M}^{2 m}$, we have the following equalities:

(i) $g(h(J X, Z), F W)+g(h(J X, W), F Z)=2(X \ln f) g$ $(Z, W)$

(ii) $g(h(X, Z), F W)+g(h(X, W), F Z)=-2(J X \ln f) g$ $(Z, W)$

for any $X \in \Gamma\left(T N_{T}^{n_{1}}\right)$ and $Z, W \in \Gamma\left(T N_{\vartheta}^{n_{1}}\right)$.

Proof. For the first part of the proof, using (9) (i) and the orthogonality of vector fields, we establish that

$$
g(h(J X, W), F Z)=-g\left(\nabla^{\sim}{ }_{W} J Z, J X\right)-g\left(\nabla^{\sim}{ }_{W} J X, P Z\right) .
$$


From the covariant derivative of the almost complex structure $J$ and from (27), we derive that

$$
\begin{aligned}
g(h(J X, W), F Z)= & (X \ln f) g(Z, W)-g\left(J X,\left(\nabla^{\sim}{ }_{W} J\right) Z\right) \\
& -(J X \ln f) g(W, P Z) .
\end{aligned}
$$

Using the structure equation (4) in the above equation and in (10) (i), we find that

$$
\begin{aligned}
g(h(J X, W), F Z)= & (X \ln f) g(Z, W)+g\left(\nabla^{\sim}{ }_{Z} F W, J X\right) \\
& -g\left(\nabla^{\sim}{ }_{Z} J X, P W\right)+g\left(\nabla^{\sim}{ }_{Z} X, W\right) \\
& -(J X \ln f) g(W, P Z) .
\end{aligned}
$$

Thus, from (27) and (10) (ii), we finally obtain

$$
g(h(J X, W), F Z)+g(h(J X, Z), F W)=2(X \ln f) g(Z, W),
$$

which is (i). Replacing $X$ wit $J X$ in (i) and using (2) (i), we obtain the required result (ii). The lemma is proven completely.

Lemma 15. Assume that $M^{n}=N_{T}^{n_{1}} \times{ }_{f} N_{\vartheta}^{n_{2}}$ is a nontrivial warped product semi-slant submanifold in a nearly Kaehler manifold; thus, we have

(i) $g(h(J X, P Z), F P W)+g(h(J X, P W), F P Z)=2(X \ln f)$ $\cos ^{2} \vartheta g(Z, W)$

(ii) $g(h(X, P Z), F P W)+g(h(X, P W), F P Z)=-2(J X \ln f)$ $\cos ^{2} \vartheta g(Z, W)$

for any $X \in \Gamma\left(T N_{T}^{n_{1}}\right)$ and $Z, W \in \Gamma\left(T N_{\vartheta}^{n_{2}}\right)$.

Proof. Replacing $Z$ and $W$ by $P Z$ and $P W$, respectively, and using (24) in Lemma 14 (i)-(ii), we directly obtain (i) and (ii), respectively. This completes the proof of the lemma.

Remark 16. In particular, if we substitute $Z=W$ in Lemma 10, then Lemma 10 coincides with Lemma 3.1 in [5].

Lemma 17. Let $M^{n}=N_{T}^{n_{1}} \times{ }_{f} N_{\vartheta}^{n_{2}}$ be a warped product semislant submanifold of a nearly Kaehler manifold $\tilde{M}^{2 m}$. Then,

(i) $g(h(X, Z), F P W)+g(h(X, W), F P Z)=(2 / 3) \cos ^{2} \vartheta$ $(X \ln f)\|Z\|^{2}$

(ii) $g(h(X, P Z), F W)+g(h(X, P W), F Z)=(2 / 3) \cos ^{2} \vartheta$ $(X \ln f)\|Z\|^{2}$

for any $X \in \Gamma\left(T N_{T}^{n_{1}}\right)$ and $Z, W \in \Gamma\left(T N_{\vartheta}^{n_{2}}\right)$.
Proof. Replacing $Z$ by $Z+W$ in Lemma 5.2 in [41], and using the linearity property of vector fields, we derive (i) and (ii). This completes the proof of the lemma.

Lemma 18. Assume that $M^{n}=N_{T}^{n_{1}} \times{ }_{f} N_{9}^{n_{2}}$ is a nontrivial warped product semi-slant submanifold in a nearly Kaehler manifold. For any $X \in \Gamma\left(T N_{T}^{n_{1}}\right)$ and $Z, W \in \Gamma\left(T N_{\vartheta}^{n_{2}}\right)$, we have

(i) $g(h(J X, Z), F P W)+g(h(J X, W), F P Z)=-(2 / 3) \cos ^{2}$ $\vartheta(J X \ln f)\|Z\|^{2}$

(ii) $g(h(J X, P Z), F W)+g(h(J X, P W), F Z)=-(2 / 3) \cos ^{2}$ $\vartheta(J X \ln f)\|Z\|^{2}$

Proof. Similarly, by replacing $X$ with $J X$ in Lemma 17 (i)-(ii), we arrive at our required results (i) and (ii) using (2).

To prove the general inequality, we require an orthonormal frame for orthonormal vector fields, as well as some preparatory results.

Lemma 19. Let $M^{n}$ be a warped product semi-slant submanifold in a nearly Kaehler manifold $\tilde{M}^{2 m}$. Thus,

(i) $g(h(X, Y), F Z)=0$

(ii) $g(h(J X, J Y), \xi)=-g(h(X, Y), \xi)$

for any $X, Y \in \Gamma\left(T N_{T}^{n_{1}}\right), Z \in \Gamma\left(T N_{\vartheta}^{n_{2}}\right)$, and $\xi \in \Gamma(v)$.

Proof. The first part of the proof is trivial; the second part can be proved in a similar manner to Lemma 5.1 in [39].

Lemma 20. Let $\varphi: M^{n}=N_{T}^{n_{1}} \times{ }_{f} N_{\vartheta}^{n_{2}} \longrightarrow \tilde{M}^{2 m}$ be an isometric immersion of a warped product semi-slant submanifold in a nearly Kaehler manifold $\tilde{M}^{2 m}$. Then, $N_{T}^{n_{1}}$ is a minimal submanifold of $\tilde{M}^{2 m}$, and the squared norm of the mean curvature of $M^{n}$ is given by

$$
\|H\|^{2}=\frac{1}{n^{2}} \sum_{r=n+1}^{2 m}\left(h_{n_{1}+1 n_{1}+1}^{r}+\cdots+h_{n n}^{r}\right)^{2}
$$

where $H$ denotes the mean curvature vector. Moreover, $n_{1}$, $n_{2}, n$, and $2 m$ are dimensions of $N_{T}^{n_{1}}, N_{\vartheta}^{n_{2}}, N_{T}^{n_{1}} \times{ }_{f} N_{\vartheta}^{n_{2}}$, and $\tilde{M}^{2 m}$, respectively.

Proof. The above lemma can be readily proven in a similar manner to Lemma 5.2 in [39].

Main inequality for warped product semi-slant submanifolds. 
Theorem 21. Let $\varphi: M^{n}=N_{T}^{n_{1}} \times{ }_{f} N_{\vartheta}^{n_{2}} \longrightarrow \tilde{M}^{2 m}$ be an isometric immersion of a warped product semi-slant submanifold $M^{n}$ in a nearly Kaehler manifold $\tilde{M}^{2 m}$. Thus,

(i) The squared norm of the second fundamental form of $M^{n}$ satisfies

$$
\|h\|^{2} \geq 2\left(n_{2}\|\nabla \ln f\|^{2}+\delta-n_{2} \Delta(\ln f)\right)
$$

where $\delta=\tilde{\tau}\left(T M^{n}\right)-\tilde{\tau}\left(T N_{T}^{n_{1}}\right)-\tilde{\tau}\left(T N_{\vartheta}^{n_{2}}\right), n_{2}$ is the dimension of the slant submanifold $N_{\vartheta}^{n_{2}}$, and $\Delta$ is the Laplacian operator of $N_{T}^{n_{1}}$.

(ii) The equality holds in (35) if and only if $N_{T}^{n_{1}}$ is totally geodesic and $N_{\vartheta}^{n_{2}}$ is totally umbilical in $\tilde{M}^{2 m}$ and if $M^{n}$ is a minimal submanifold of $\tilde{M}^{2 m}$

Proof. The proof proceeds in a similar manner to the proof of Theorem 29 [31] if we consider the nearly Kaehler manifold, instead of nearly trans-Sasakian manifold.

3.1. Applications of Theorem 21 to Generalized Complex Space Forms. In this section, we prove our main theorem using Theorem 21 for a generalized complex space form. Then, we give the following result.

Theorem 22. Assume that $\varphi: M^{n}=N_{T}^{n_{1}} \times{ }_{f} N_{\vartheta}^{n_{2}} \longrightarrow \tilde{M}^{2 m}$ $(\kappa, \alpha)$ be an isometric immersion of a warped product semislant $N_{T}^{n_{1}} \times{ }_{f} N_{\vartheta}^{n_{2}}$ into generalized complex space form $\tilde{M}^{2 m}$ $(\kappa, \alpha)$ admitting a nearly Kaehler structure with constant holomorphic sectional curvature $\kappa$ of constant type $\alpha$. Then,

(i) The squared norm of the second fundamental form of $M^{n}$ satisfies

$$
\|h\|^{2} \geq 2 n_{2}\left\{\left(\frac{\kappa+3 \alpha}{4}\right) n_{1}-\Delta(\ln f)+\|\nabla \ln f\|^{2}\right\}
$$

where $n_{1}=\operatorname{dim} N_{T}, n_{2}=\operatorname{dim}_{\mathbf{R}} N_{\vartheta}, n=\operatorname{dim} N_{T} \times{ }_{f} N_{\vartheta}$ and 2 $m=\operatorname{dim} \tilde{M}^{2 m}(\kappa, \alpha)$.

(ii) The equality holds in (36), if and only if $N_{9}^{n_{2}}$ and $N_{T}^{n_{2}}$ are totally umbilical and totally geodesic submanifolds in $\tilde{M}$, respectively. Moreover, $M^{n}$ is a minimal submanifold of $\tilde{M}^{2 m}(\kappa, \alpha)$
Proof. Letting us substitute $X=W=e_{i}$ and $Y=Z=e_{j}$ in (8), we get

$$
\begin{aligned}
\tilde{R}\left(e_{i}, e_{j}, e_{j}, e_{i}\right)= & \frac{\kappa+3 \alpha}{4}\left\{g\left(e_{i}, e_{i}\right) g\left(e_{j}, e_{j}\right)-g\left(e_{i}, e_{j}\right) g\left(e_{i}, e_{j}\right)\right\} \\
& +\frac{\kappa-\alpha}{4}\left\{g\left(e_{i}, J e_{j}\right) g\left(J e_{j}, e_{i}\right)\right. \\
& \left.-g\left(e_{i}, J e_{i}\right) g\left(e_{j}, J e_{j}\right)+2 g^{2}\left(J e_{j}, e_{i}\right)\right\} .
\end{aligned}
$$

Taking the summation over the basis vector fields of $T$ $M^{n}$ such that $1 \leq i \neq j \leq n$, one shows that

$$
2 \tilde{\tau}\left(T M^{n}\right)=\left(\frac{c+3 \alpha}{4}\right) n(n-1)+3\left(\frac{c-\alpha}{4}\right) \sum_{1 \leq i \neq j \leq n} g^{2}\left(P e_{i}, e_{j}\right) .
$$

$M^{n}$ is a warped product of holomorphic and proper slant submanifolds in a generalized complex space form $\tilde{M}^{2 m}(c, \alpha)$. Thus, we set the following frame of orthonormal vector fields as

$$
\begin{aligned}
e_{1}, e_{2} & =J e_{1}, \cdots e_{2 d_{1}-1}, e_{2 d_{1}}=J e_{2 d_{1}-1}, e_{2 d_{1}+1}, e_{2 d_{1}+2} \\
& =\sec \vartheta P e_{2 d_{1}+1}, \cdots e_{2 d_{1}+2 d_{2}-1} e_{2 d_{1}+2 d_{2}}=\sec \vartheta P e_{d_{1}-1}
\end{aligned}
$$

From using the above orthonormal frame, we obtain

$$
\begin{aligned}
g^{2}\left(J e_{i}, e_{i+1}\right) & =1, \text { for } i \in\left\{1 \cdots n_{1}-1\right\} \\
& =\cos ^{2} \vartheta \text { for } i \in\left\{n_{1}+1, \cdots n_{1}+n_{2}-1\right\}
\end{aligned}
$$

Thus, it is easily seen that

$$
\sum_{i, j=1}^{n} g^{2}\left(P e_{i}, e_{j}\right)=n_{1}+n_{2} \cos ^{2} \vartheta
$$

From (38) and (40), it follows that

$$
2 \tilde{\tau}\left(T M^{n}\right)=\left(\frac{\kappa+3 \alpha}{4}\right) n(n-1)+3\left(\frac{\kappa-\alpha}{4}\right)\left\{n_{1}+n_{2} \cos ^{2} \vartheta\right\} .
$$

Similarly, for $T N_{T}^{n_{1}}$, we derive

$$
2 \tilde{\tau}\left(T N_{T}^{n_{1}}\right)=\left(\frac{\kappa+3 \alpha}{4}\right) n_{1}\left(n_{1}-1\right)+3\left(\frac{\kappa-\alpha}{4}\right) n_{1} .
$$

Now using fact that $\|P\|^{2}=n_{2} \cos ^{2} \vartheta$, for slant bundle $T N_{\vartheta}^{n_{2}}$, one derives

$$
2 \tilde{\tau}\left(T N_{\vartheta}^{n_{2}}\right)=\left(\frac{\kappa+3 \alpha}{4}\right) n_{2}\left(n_{2}-1\right)+3\left(\frac{\kappa-\alpha}{4}\right) n_{2} \cos ^{2} \vartheta
$$

Therefore, substituting (41), (42), and (40) in Theorem 
21 , we get the required result (29). The equality case follows from Theorem 21 (ii). Thus, the proof is complete.

Notably, the following corollary can be readily obtained in terms of the Hessian tensor of the warping function $\ln f$ for a warped product submanifold.

Corollary 23. Let $\varphi: M^{n}=N_{T}^{n_{1}} \times{ }_{f} N_{\vartheta}^{n_{2}} \longrightarrow \tilde{M}^{2 m}(\kappa, \alpha)$ be an isometric immersion of a warped product $N_{T}^{n_{1}} \times{ }_{f} N_{9}^{n_{2}}$ into generalized complex space form $\tilde{M}^{2 m}(\kappa, \alpha)$ admitting a nearly Kaehler structure. Then,

$$
\|h\|^{2} \geq 2 n_{2}\left(\left(\frac{\kappa+3 \alpha}{4}\right) n_{1}+\|\nabla \ln f\|^{2}+\operatorname{Trace}\left(H^{\ln f}\right)\right)
$$

where $H^{\ln f}$ is the Hessian tensor of the warping function $\ln f$.

\section{Compact-Orientated Warped Product semi- slant Submanifolds}

In this section, we consider compact Riemannian manifolds without boundaries; that is, $\partial M^{n}=\varnothing$. Applying these to warped product semi-slant submanifolds, and using integration theory on the manifold, we obtain several characterizations.

Theorem 24. Let $M^{n}=N_{T}^{n_{1}} \times{ }_{f} N_{\vartheta}^{n_{2}}$ be a compact-orientated warped product semi-slant submanifold generalized complex space form $\tilde{M}^{2 m}(\kappa, \alpha)$ admitting a nearly Kaehler structure. Then, $M^{n}$ is a trivial warped product if and only if

$$
\|h\|^{2} \geq 2\left(\frac{\kappa+3 \alpha}{4}\right) n_{2} n_{1}
$$

Proof. From Theorem 21, we have

$$
\|h\|^{2} \geq 2\left(\frac{\kappa+3 \alpha}{4}\right) n_{2} n_{1}-n_{2} \Delta(\ln f)+n_{2}\|\nabla \ln f\|^{2} .
$$

This implies that

$$
n_{2}\|\nabla \ln f\|^{2}+2\left(\frac{\kappa+3 \alpha}{4}\right) n_{2} n_{1}-\|h\|^{2} \leq n_{2} \Delta(\ln f) .
$$

Applying integration theory on the compact-orientated Riemannian manifold $M^{n}$ without boundary, and then using (21), we obtain

$\int_{M^{n}}\left(2\left(\frac{\kappa+3 \alpha}{4}\right) n_{2} n_{1}+n_{2}\|\nabla \ln f\|^{2}-\|h\|^{2}\right) \mathrm{d} V \leq \int_{M^{n}} \Delta(\ln f) \mathrm{d} V=0$.

Now, if the inequality (44) holds, then from (47), we find that

$$
\int_{M^{n}}\left(\|\nabla \ln f\|^{2}\right) d V \leq 0
$$

which is impossible for a positive integrable function; hence, $\nabla \ln f=0$; that is, $f$ is a constant function on $M^{n}$. Thus, by Remark 11 on warped product manifolds, $M^{n}$ is trivial. The converse proof is straightforward.

To prove the equality case, we must prove the following theorem for later use.

Theorem 25. Let $\varphi$ be a $\mathscr{D}^{9}$-minimal isometric immersion of a warped product semi-slant submanifold $N_{T}^{n_{1}} \times{ }_{f} N_{\vartheta}^{n_{2}}$ in a nearly Kaehler manifold $\tilde{M}^{2 m}$. If $N_{9}^{n_{2}}$ is totally umbilical in $\tilde{M}^{2 m}$, then $\phi$ is $N_{\vartheta}^{n_{2}}$-totally geodesic.

Proof. Let us assume that the second fundamental forms of $M^{n}$ and $\tilde{M}^{2 m}$ are denoted by $h^{*}$ and $\tilde{h}$, respectively; we define

$$
h(Z, W)+h^{*}(Z, W)=\tilde{h}(Z, W) .
$$

for any vector fields $Z$ and $W$ that are tangential to $N_{9}^{n_{2}}$. Thus, the above hypothesis and Remark 12 show that $N_{\vartheta}^{n_{2}}$ is totally umbilical in $\tilde{M}^{2 m}$, owing to its being totally umbilical in $M^{n}$. Then, following Lemma 10 (ii), equation (47) can be written as

$$
h(Z, W)=g(Z, W)(\xi+\nabla(\ln f))
$$

where the vector field $\xi$ is normal to $\Gamma\left(T N_{\vartheta}\right)$ and is such that $\xi \in \Gamma\left(T M^{n}\right)$. Assuming that $\left\{e_{1}^{*}, \cdots e_{n_{2}}^{*}\right\}$ is an orthonormal frame of the slant submanifold $N_{9}^{n_{2}}$, then by taking a summation over the vector fields of $N_{\vartheta}^{n_{2}}$ in equation (49), we obtain

$$
\sum_{i, j=1}^{n_{2}} h\left(e_{i}^{*}, e_{j}^{*}\right)=(\xi+\nabla(\ln f)) \sum_{i, j=1}^{n_{2}} g\left(e_{i}^{*}, e_{j}^{*}\right)
$$

The left-hand side of the above equation identically vanishes due to the $\mathscr{D}^{9}$-minimality of $\varphi$, such that $\sum_{i, j=1}^{n_{2}} h$ $\left(e_{i}^{*}, e_{j}^{*}\right)=0$. Then, equation (50) takes the following form:

$$
n_{2}(\xi+\nabla(\ln f))=0
$$

This implies that $N_{\vartheta}^{n_{2}}$ is nonempty, such that

$$
\xi=-\nabla(\ln f) .
$$

Thus, from (50) and (53), it follows that $h(Z, W)=0$, for every $Z, W \in \Gamma\left(T N_{9}^{n_{2}}\right)$. This means that $\varphi$ is $N_{9}^{n_{2}}$-totally geodesic. This completes the proof of the theorem.

Theorem 26. Let $M^{n}=N_{T}^{n_{1}} \times{ }_{f} N_{\vartheta}^{n_{2}}$ be a compact-orientated warped product semi-slant submanifold in a generalized 
complex space form $\tilde{M}^{2 m}(\kappa, \alpha)$ admitting a nearly Kaehler structure. Then, $M^{n}$ is a trivial warped product if and only if

$$
\sum_{i=1}^{n_{1}} \sum_{j=1}^{n_{2}}\left\|h_{v}\left(e_{i}, e_{j}\right)\right\|^{2}=\left(\frac{\kappa+3 \alpha}{4}\right) n_{2} n_{1}
$$

where $n_{1}=\operatorname{dim} N_{T}$ and $n_{2}=\operatorname{dim}_{\mathbf{R}} N_{\vartheta}$. Moreover, $h_{v}$ is a component of $h$ in $\Gamma(v)$.

Proof. We assume that the equality sign holds in (36); then, we have

$$
\begin{aligned}
\left(\frac{\kappa+3 \alpha}{4}\right) n_{2} n_{1}+2 n_{2}\|\nabla \ln f\|^{2}= & \|h(\mathscr{D}, \mathscr{D})\|^{2}+\left\|h\left(\mathscr{D}^{9}, \mathscr{D}^{9}\right)\right\|^{2} \\
& +2\left\|h\left(\mathscr{D}, \mathscr{D}^{9}\right)\right\|^{2}+2 n_{2} \Delta(\ln f) .
\end{aligned}
$$

However, the equality case of inequality (36) implies that $N_{T}^{n_{1}}$ is totally geodesic in a nearly Kaehler manifold; this means that $h\left(e_{i}, e_{j}\right)=0$, for any $1 \leq i, j \leq 2 d_{1}$. Moreover, $N_{9}^{n_{2}}$ is totally umbilical and can be written as

$$
h\left(e_{t}^{*}, e_{s}^{*}\right)=g\left(e_{t}^{*}, e_{s}^{*}\right) H,
$$

for any $1 \leq t, s \leq 2 d_{2}$. Furthermore, $M^{n}$ is a minimal submanifold of a nearly Kaehler manifold; thus, its mean curvature vector $H$ should be zero; that is, $H=0$; hence, $h\left(e_{t}^{*}, e_{s}^{*}\right)=0$, for every $1 \leq t, s \leq 2 d_{2}$ through Theorem 25

$$
\left(\frac{\kappa+3 \alpha}{4}\right) n_{2} n_{1}=n_{2} \Delta(\ln f)+\left\|h\left(\mathscr{D}, \mathscr{D}^{9}\right)\right\|^{2}-n_{2}\|\nabla \ln f\|^{2} .
$$

We assume that $M^{n}$ is a compact submanifold; thus, $M^{n}$ is closed and bounded; hence, by integrating the above equation over the volume element $\mathrm{d} V$ of $M^{n}$ and using (21), we find that

$$
\int_{M^{n}}\left\{\left\|h\left(\mathscr{D}, \mathscr{D}^{9}\right)\right\|^{2}-n_{2}\|\nabla \ln f\|^{2}\right\} \mathrm{d} V=\int_{M^{n}}\left(\frac{\kappa+3 \alpha}{4}\right) n_{2} n_{1} d V .
$$

Now, let $X=e_{i}$ and $Z=e_{j}$ for $1 \leq i \leq n_{1}$ and $1 \leq j \leq n_{2}$, respectively; then, using (14) and expressing in terms of the orthonormal frame, we have

$$
\left\|h\left(\mathscr{D}, \mathscr{D}^{9}\right)\right\|^{2}=\sum_{k=1}^{2 m} \sum_{i=1}^{n_{1}} \sum_{j=1}^{n_{1}} g\left(h\left(e_{i}, e_{j}^{*}\right), e_{k}\right)^{2} .
$$

In the above equation, the first term on the right-hand side is the $F D^{9}$-component and the second term is the $v$ -component. Let us suppose that $M^{n}=N_{T}^{n_{1}} \times{ }_{f} N_{9}^{n_{2}}$ is a warped product semi-slant submanifold of an $n$-dimension in a nearly Kaehler manifold $\tilde{M}^{2 m}$ of $2 m$ dimensions, such that $\operatorname{dim} N_{T}=n_{1}=2 d_{1}$ and $\operatorname{dim}_{\mathbf{R}} N_{\vartheta}=n_{2}=2 d_{2}$. We assume that the tangent spaces of $N_{T}^{n_{1}}$ and $N_{\vartheta}^{n_{2}}$ are $\mathscr{D}$ and $\mathscr{D}^{9}$, respectively. We further assume that $\left\{e_{1}, e_{2}, \cdots\right.$, $\left.e_{d_{1}}, e_{d_{1}+1}=J e_{1}, \cdots, e_{2 d_{1}}=J e_{d_{1}}\right\}$ is a local orthonormal frame of $T N_{T}^{n_{1}}$ and that $\left\{e_{2 d_{1}+1}=e_{1}^{*}, \cdots, e_{2 d_{1}+d_{2}}=e_{d_{2}}^{*}, e_{2 d_{1}+d_{2}+1}=\right.$ $\left.e_{d_{2}+1}^{*}=\sec \vartheta P e_{1}^{*}, \cdots, e_{n_{1}+n_{2}}=e_{n_{2}}^{*}=\sec \vartheta P e_{d_{2}}^{*}\right\}$ is a local orthonormal frame of $T N_{\vartheta}^{n_{2}}$. Thus, the orthonormal frames of the normal subbundles $F D^{9}$ and $v$ are $\left\{e_{n+1}=\tilde{e}_{1}=\csc 9 F\right.$ $e_{1}^{*}, \cdots, e_{n+d_{2}}=\tilde{e}_{d_{2}}=\csc \vartheta F e_{1}^{*}, e_{n+d_{2}+1}=\tilde{e}_{d_{2}+1}=\csc \vartheta \sec \vartheta F P e_{1}^{*}$, $\left.\cdots, e_{n+2 d_{2}}=\tilde{e}_{2 d_{2}}=\csc \vartheta \sec \vartheta F P e_{d_{2}}^{*}\right\}$ and $\left\{e_{n+2 d_{2}+1}, \cdots, e_{2 m}\right\}$, respectively. Taking a summation over the vector fields on $N_{T}^{n_{1}}$ and $N_{\vartheta}^{n_{2}}$ and using adapted frame fields, we obtain

$$
\begin{aligned}
\left\|h\left(\mathscr{D}, \mathscr{D}^{9}\right)\right\|^{2}= & \operatorname{ssc}^{2} \vartheta \sum_{i=1}^{d_{1}} \sum_{j, k=1}^{d_{2}} g\left(h\left(e_{i}, e_{j}^{*}\right), F e_{k}^{*}\right)^{2} \\
& +\csc ^{2} \vartheta \sec ^{2} \vartheta \sum_{i=1}^{d_{1}} \sum_{j, k=1}^{d_{2}} g\left(h\left(e_{i}, P e_{j}^{*}\right), F e_{k}^{*}\right)^{2} \\
& +\csc ^{2} \vartheta \sec ^{2} \vartheta \sum_{i=1}^{d_{1}} \sum_{j, k=1}^{d_{2}} g\left(h\left(J e_{i}, e_{j}^{*}\right), F P e_{k}^{*}\right)^{2} \\
& +\csc ^{2} \vartheta \sec ^{2} \vartheta \sum_{i=1}^{d_{1}} \sum_{j, k=1}^{d_{2}} g\left(h\left(J e_{i}, e_{j}^{*}\right), F P e_{k}^{*}\right)^{2} \\
& +\csc ^{2} \vartheta \sec ^{4} \vartheta \sum_{i=1}^{d_{1}} \sum_{j, k=1}^{d_{2}} g\left(h\left(J e_{i}, P e_{j}^{*}\right), F P e_{k}^{*}\right)^{2} \\
& +\csc ^{2} \vartheta \sec ^{2} \vartheta \sum_{i=1}^{d_{1}} \sum_{j, k=1}^{d_{2}} g\left(h\left(J e_{i}, P e_{j}^{*}\right), F e_{k}^{*}\right)^{2} \\
& +\csc ^{2} \vartheta \sum_{i=1}^{d_{1}} \sum_{j, k=1}^{d_{2}} g\left(h\left(J e_{i}, e_{j}^{*}\right), F e_{k}^{*}\right)^{2} \\
& +\csc ^{2} \vartheta \sec ^{4} \vartheta \sum_{i=1}^{d_{1}} \sum_{j, k=1}^{d_{2}} g\left(h\left(e_{i}, P e_{j}^{*}\right), F P e_{r}^{*}\right)^{2} \\
& +\sum_{r=n+n_{2}+1}^{2 m} \sum_{i=1}^{n_{1}} \sum_{j=1}^{n_{2}} g\left(h\left(e_{i}, e_{j}\right), e_{r}\right)^{2} .
\end{aligned}
$$

Then, using Lemmas 14 to 18 in the above equations, we derive

$$
\begin{aligned}
\left\|h\left(\mathscr{D}, \mathscr{D}^{9}\right)\right\|^{2}= & 2\left(\csc ^{2} \vartheta+\frac{1}{9} \cot ^{2} \vartheta\right) \sum_{i=1}^{d_{1}} \sum_{j=1}^{d_{2}}\left(e_{i} \ln f\right)^{2} g\left(e_{j}^{*}, e_{j}^{*}\right)^{2} \\
& +2\left(\csc ^{2} \vartheta+\frac{1}{9} \cot ^{2} \vartheta\right) \sum_{i=1}^{d_{1}} \sum_{j=1}^{d_{2}}\left(J e_{i} \ln f\right)^{2} g\left(e_{j}^{*}, e_{j}^{*}\right)^{2} \\
& +\sum_{r=n+n_{2}+1}^{2 m} \sum_{i=1}^{n_{1}} \sum_{j=1}^{n_{2}} g\left(h\left(e_{i}, e_{j}\right), e_{r}\right)^{2} .
\end{aligned}
$$


From (17), the last equation can be expressed as

$$
\begin{aligned}
\left\|h\left(\mathscr{D}, \mathscr{D}^{\vartheta}\right)\right\|^{2}= & 2\left(\csc ^{2} \vartheta+\frac{1}{9} \cot ^{2} \vartheta\right)\|\nabla \ln f\|^{2} \sum_{j=1}^{d_{2}} g\left(e_{j}^{*}, e_{j}^{*}\right)^{2} \\
& +\sum_{r=n+n_{2}+1}^{2 m} \sum_{i=1}^{n_{1}} \sum_{j=1}^{n_{2}} g\left(h\left(e_{i}, e_{j}\right), e_{r}\right)^{2}
\end{aligned}
$$

which implies that

$$
\left\|h\left(\mathscr{D}, \mathscr{D}^{9}\right)\right\|^{2}=n_{2}\left(1+\frac{10}{9} \cot ^{2} \vartheta\right)\|\nabla \ln f\|^{2}+\sum_{i=1}^{n_{1}} \sum_{j=1}^{n_{2}}\left\|h_{v}\left(e_{i}, e_{j}\right)\right\|^{2} .
$$

Then, from (58) and (63), it follows that

$\int_{M^{n}}\left\{\sum_{i=1}^{n_{1}} \sum_{j=1}^{n_{2}}\left\|h_{\mu}\left(e_{i}, e_{j}\right)\right\|^{2}+\frac{10}{9} n_{2} \cot ^{2} \vartheta\|\nabla \ln f\|^{2}\right\} \mathrm{d} V=\int_{M^{n}}\left(\frac{\kappa+3 \alpha}{4}\right) n_{2} n_{1} d V$

If (54) holds identically, then from (66), we find that either $f$ is constant on $M^{n}$ or $\cot \vartheta=0$. However, $M^{n}$ is a proper semi-slant submanifold; thus, $M^{n}$ is a simple Riemannian product. The converse proof follows immediately from (66). Hence, the theorem is proven completely.

Corollary 27. Assume that $M^{n}=N_{T}^{n_{1}} \times{ }_{f} N_{\vartheta}^{n_{2}}$ is a warped product semi-slant submanifold in a generalized complex space form $\tilde{M}^{2 m}(\kappa, \alpha)$ admitting a nearly Kaehler structure. Suppose that $N_{T}^{n_{1}}$ is a compact invariant submanifold and $\lambda_{T}$ is a nonzero eigenvalue of the Laplacian on $N_{T}^{n_{1}}$. Then,

$\int_{N_{T}^{n_{1}}}\|h\|^{2} d V_{T} \geq \int_{N_{T}^{n_{1}}}\left(\frac{\kappa+3 \alpha}{4}\right) n_{2} n_{1} d V+2 n_{2} \lambda_{T} \int_{N_{T}^{n_{1}}}(\ln f)^{2} d V_{T}$,

where $d V_{T}$ is the volume element on $N_{T}^{n_{1}}$. The equality sign holds if and only if the following are satisfied:

(i) $\nabla \ln f=\lambda_{T} \ln f$

(ii) A warped product semi-slant submanifold $M^{n}=N_{T}^{n_{1}}$ $\times{ }_{f} N_{\vartheta}^{n_{2}}$ is both $N_{T}^{n_{1}}$ - and $N_{\vartheta}^{n_{2}}$-totally geodesic

Proof. Thus, using the minimum principle property, we have

$$
\int_{N_{T}^{n_{1}}}\|\nabla \ln f\|^{2} d V_{T} \geq \lambda_{T} \int_{N_{T}^{n_{1}}}(\ln f)^{2} d V_{T}
$$

The equality holds if and only if one has $\nabla \ln f=\lambda_{T} \ln f$. Thus, from (44) and (66), we require the result (65). Similarly, it is clear that the equality sign of (65) holds identically if and only if the warped product is both $N_{T}^{n_{1}}$ - and $N_{\vartheta}^{n_{2}}$ -totally geodesic. This completes the proof of the corollary.

Corollary 28. Let $M^{n}=N_{T}^{n_{1}} \times{ }_{f} N_{\vartheta}^{n_{2}}$ be a warped product semi-slant submanifold in a generalized complex space form $\tilde{M}^{2 m}(\kappa, \alpha)$ admitting a nearly Kaehler structure such that $N_{T}^{n_{1}}$ is compact, and let $\lambda_{T}$ be a nonzero eigenvalue of the Laplacian on $N_{T}^{n_{1}}$. Then,

$$
\begin{aligned}
\int_{N_{T}^{n_{1}}}\left(\sum_{i=1}^{n_{1}} \sum_{j=1}^{n_{2}}\left\|h_{v}\left(e_{i}, e_{j}\right)\right\|^{2}\right) d V_{T} \geq & \int_{N_{T}^{n_{I}}} 2\left(\frac{\kappa+3 \alpha}{4}\right) n_{2} n_{1} d V \\
& +\frac{10}{9} n_{2} \cot ^{2} \vartheta \lambda_{T} \int_{N_{T}^{n_{I}}}(\ln f)^{2} d V_{T}
\end{aligned}
$$

The equality sign holds if and only if the following are satisfied:

(a) $\nabla \ln f=\lambda_{T} \ln f$

(b) A warped product semi-slant submanifold $M^{n}=N_{T}^{n_{1}}$ $\times{ }_{f} N_{\vartheta}^{n_{2}}$ is both $N_{T}^{n_{1}}$ - and $N_{\vartheta}^{n_{2}}$-totally geodesic

Proof. The proof follows from (67) and (66). This completes the proof of the theorem.

\section{Applications to Dirichlet Energy Functions and Hamiltonian}

We discuss connected, compact Riemannian manifolds with borders in this section; that is, $\partial M \neq \varnothing$. Using Hopf's lemma, we apply these to warped product submanifolds. To determine whether nontrivial warped products become trivial warped product submanifolds of nearly Kaehler manifolds, we obtain necessary and sufficient conditions in terms of Dirichlet energy (analogous to kinetic energy) and Hamiltonian of warping functions.

Theorem 29. Assume that $\varphi: M^{n}=N_{T}^{n_{1}} \times{ }_{f} N_{\vartheta}^{n_{2}}$ is an isometric immersion of a warped product semi-slant in a generalized complex space form $\tilde{M}^{2 m}(\kappa, \alpha)$ admitting a nearly Kaehler structure. A connected and compact warped product $N_{T}^{n_{1}} \times_{f}$ $N_{\vartheta}^{n_{2}}$ is trivial if and only if the Dirichlet energy function satisfies

$E(\ln f)=\frac{9}{20 n_{2}} \tan ^{2} \vartheta \int_{M^{n}}\left\{\left(\frac{\kappa+3 \alpha}{4}\right) n_{2} n_{1}-\sum_{i=1}^{n_{1}} \sum_{j=1}^{n_{2}}\left\|h_{v}\left(e_{i}, e_{j}\right)\right\|^{2}\right\} d V$,

where $E(\ln f)$ represents the Dirichlet energy of the warping function $\ln f$ and $d V$ is the volume element on $M^{n}$. 
Proof. Combining equations (57) and (63), we obtain

$$
\left(\frac{\kappa+3 \alpha}{4}\right) n_{2} n_{1}=n_{2} \Delta(\ln f)+\sum_{i=1}^{n_{1}} \sum_{j=1}^{n_{2}}\left\|h_{v}\left(e_{i}, e_{j}\right)\right\|^{2}+n_{2} \frac{10}{9} \cot ^{2} \vartheta\|\nabla \ln f\|^{2}
$$

Taking an integration on $M^{n}$ over the volume element $d V$ with a nonempty boundary in the above equation, we find that

$$
\begin{aligned}
\int_{M^{n}}\left(\frac{\kappa+3 \alpha}{4}\right) n_{2} n_{1} d V= & n_{2} \int_{M^{n}}(\Delta(\ln f)) \mathrm{d} V \\
& +\int_{M^{n}}\left(\sum_{i=1}^{n_{1}} \sum_{j=1}^{n_{2}}\left\|h_{v}\left(e_{i}, e_{j}\right)\right\|^{2}\right) \mathrm{d} V \\
& +\frac{10}{9} n_{2} \cot ^{2} \vartheta \int_{M^{n}}\left(\|\nabla \ln f\|^{2}\right) d V
\end{aligned}
$$

Then, from (23) and (70), it follows that

$$
\begin{aligned}
\frac{1}{n_{2}} \int_{M^{n}}\left(\frac{\kappa+3 \alpha}{4}\right) n_{2} n_{1} d V= & \int_{M^{n}} \Delta(\ln f) \mathrm{d} V \\
& +\frac{1}{n_{2}} \int_{M^{n}}\left(\sum_{i=1}^{n_{1}} \sum_{j=1}^{n_{2}}\left\|h_{v}\left(e_{i}, e_{j}\right)\right\|^{2}\right) \mathrm{d} V \\
& +\frac{20}{9} \cot ^{2} \vartheta E(\ln f) .
\end{aligned}
$$

Equality (68) is satisfied if and only if we obtain from (71) the condition that $\int_{M^{n}} \Delta(\ln f) \mathrm{d} V=0$, which implies that $\Delta(\ln f)=0$. The theorem hypothesizes $M^{n}$ as a connected, compact warped product semi-slant submanifold; thus, Theorem 4 implies that $\ln f=0 \Longrightarrow f=1$, which means that $f$ is constant on $M^{n}$. Thus, the theorem is proven completely.

In a similar manner, we derive several characterizations in terms of the Hamiltonian.

Theorem 30. Let $M^{n}=N_{T}^{n_{1}} \times{ }_{f} N_{\vartheta}^{n_{2}}$ be a connected and compact warped product semi-slant submanifold in a generalized complex space form $\tilde{M}^{2 m}(\kappa, \alpha)$ admitting a nearly Kaehler structure. Then, $M^{n}$ is a trivial warped product submanifold of $N_{T}^{n_{1}}$ and $N_{\vartheta}^{n_{2}}$ if and only if the Hamiltonian of the warping function satisfies the following equality:

$H(d(\ln f), x)=\frac{9}{20 n_{2}} \tan ^{2} \vartheta\left(\left(\frac{\kappa+3 \alpha}{4}\right) n_{2} n_{1}-\frac{1}{n_{2}} \sum_{i=1}^{n_{1}} \sum_{j=1}^{n_{2}}\left\|h_{v}\left(e_{i}, e_{j}\right)\right\|^{2}\right)$.
Proof. Using (15) in (69), we derive

$$
\frac{20}{9} \cot ^{2} \vartheta H(\mathrm{~d}(\ln f), x)+\Delta(\ln f)=\left(\frac{\kappa+3 \alpha}{4}\right) n_{1}-\frac{1}{n_{2}} \sum_{j=1}^{n_{2}}\left\|h_{v}\left(e_{i}, e_{j}\right)\right\|^{2}
$$

Equation (72) is obtained if and only if $\Delta(\ln f)=0$ on $M^{n}$; thus, by Theorem 4 , the warped product submanifold $M^{n}$ is trivial. This completes the proof of the theorem. below.

The analogy of Theorem 3 for this case is classified

Theorem 31. Assume that $\varphi: M^{n}=N_{T}^{n_{1}} \times{ }_{f} N_{\vartheta}^{n_{2}}$ is an isometric immersion of a compact warped product semi-slant submanifold in a generalized complex space form $\tilde{M}^{2 m}(\kappa, \alpha)$ admitting a nearly Kaehler structure. Let the warping function be the solution of the Euler-Lagrange equation; then, $M^{n}$ is necessarily a trivial warped product if

$$
\|h\|^{2} \geq 2\left(\frac{\kappa+3 \alpha}{4}\right) n_{2} n_{1}
$$

Proof. If the warping function satisfies the conditions of the Euler-Lagrange equation, then from Theorem 3, we obtain

$$
\Delta(\ln f)=0
$$

Thus, from (44) and (75), we derive

$$
\|h\|^{2} \geq 2\left(\frac{\kappa+3 \alpha}{4}\right) n_{2} n_{1}+n_{2}\|\nabla \ln f\|^{2}
$$

Suppose that inequality (74) holds; then, (76) implies that the warping function must be constant on $M^{n}$. This completes the proof of the theorem.

Theorem 32. Assume that $\varphi: M^{n}=N_{T}^{n_{1}} \times{ }_{f} N_{\vartheta}^{n_{2}}$ is an isometric immersion of a compact warped product semi-slant submanifold in a generalized complex space form $\tilde{M}^{2 m}(\kappa, \alpha)$ admitting a nearly Kaehler structure and that the warping function is a solution of the Euler-Lagrange equation. Then, the necessary and sufficient conditions for the warped product $N_{T}^{n_{1}} \times{ }_{f} N_{9}^{n_{2}}$ being trivial are as follows:

$$
\sum_{i=1}^{n_{1}} \sum_{j=1}^{n_{2}}\left\|h_{v}\left(e_{i}, e_{j}\right)\right\|^{2}=\left(\frac{\kappa+3 \alpha}{4}\right) n_{2} n_{1}
$$

Proof. The proof of the above theorem is the same as that of Theorem 31; it uses (57), (63), and Theorem 3. This completes the proof of the theorem. 


\section{Classification of Ricci Curvature and Divergence of the Hessian Tensor}

In this section, we study several applications of the derived inequality by considering equality cases. Let us identify any $(0,2)$-tensor $T$ on $M$ with a $(1,1)$-tensor via

$$
g(T(Z), Y)=T(Z, Y)
$$

for all $Y, Z \in \Gamma(T M)$. Thus, we obtain

$$
\operatorname{div}(\phi T)=\phi \operatorname{div} T+T(\nabla \phi, \cdot), \quad \nabla(\phi T)=\phi \nabla T+d \phi \otimes T,
$$

for all $\phi \in C^{\infty}(M)$. In particular, we have $\operatorname{div}(\phi g)=d \phi$. Moreover, the following general facts are well established in the literature:

$$
\text { (1) } \operatorname{div} \nabla^{2} \phi=\operatorname{Ric}(\nabla \phi, \cdot)+d \Delta \phi, \quad \text { (ii) } \frac{1}{2} d\|\nabla\|^{2}=\nabla^{2} \phi(\nabla, \cdot) \text {. }
$$

We consider $M^{n}$ to be a compact Riemannian manifold with boundaries and obtain the following classification results.

Theorem 33. Assume that $\varphi: M^{n}=N_{T}^{n_{1}} \times{ }_{f} N_{\vartheta}^{n_{2}}$ is an isometric immersion of a compact warped product semi-slant submanifold $N_{T}^{n_{1}} \times{ }_{f} N_{\vartheta}^{n_{2}}$ in a generalized complex space form $\tilde{M}^{2 m}(\kappa, \alpha)$ admitting a nearly Kaehler structure. If

$$
\sum_{i=1}^{n_{1}} \sum_{j=1}^{n_{2}}\left\|h_{v}\left(e_{i}, e_{j}^{*}\right)\right\|^{2}=\left(\frac{\kappa+3 \alpha}{4}\right) n_{2} n_{1}+n_{2} \int_{M^{n}} \mathscr{R} i c(\nabla \ln f, \cdot) d V,
$$

is satisfied for the warped product submanifold $M^{n}$, then at least one of the following statements is true for $M^{n}$ :

(i) The warped product semi-slant submanifold $N_{T}^{n_{1}} \times{ }_{f}$ $\mathrm{N}_{\vartheta}^{n_{2}}$ becomes a CR-warped product that is isometrically immersed in a nearly Kaehler manifold

(ii) The nontrivial warped product semi-slant submanifold $N_{T}^{n_{1}} \times{ }_{f} N_{\vartheta}^{n_{2}}$ in a nearly Kaehler manifold is a simple Riemannian product of $N_{T}^{n_{1}}$ and $N_{\vartheta}^{n_{2}}$

Proof. Using the first identity of (79) and setting $\phi=\ln f$, we derive

$$
\operatorname{div} \nabla^{2} \ln f=\operatorname{Ric}(\nabla \ln f, \cdot)+d \Delta(\ln f),
$$

from the hypothesis of the theorem; assuming that $M^{n}$ is a compact warped product submanifold with a boundary and integrating along the volume element $\mathrm{dV}$, we obtain

$$
\Delta(\ln f)=\int_{M}\left(\operatorname{div} \nabla^{2} \ln f\right) \mathrm{dV}-\int_{M} \mathscr{R} i c(\nabla \ln f, \cdot) \mathrm{dV}
$$

We use the Green theorem on the compact manifold $M^{n}$; given a smooth function $f: M \longrightarrow \mathbb{R}$, we have $\int_{M} \Delta$ $f d V=0$. We can apply the results of Yano and Kon (see [33]) immediately as $\Delta f=-\operatorname{div}(\nabla f)$. From the Green lemma, $\int_{M} \operatorname{div}(X) \mathrm{dV}=0$ for any arbitrary vector field $X$ on $M^{n}$. Thus, we obtain $\int_{M}\left(\operatorname{div} \nabla^{2} \ln f\right) \mathrm{dV}=0 ; \nabla^{2} \ln f$ is the Hessian tensor of the warped function (or the Laplacian of $\ln f$ ); hence, (82) implies that

$$
\Delta(\ln f)=-\int_{M} \mathscr{R} i c(\nabla \ln f, \cdot) \mathrm{dV}
$$

Meanwhile, if we assume that the equality holds in the inequality (36), then from (57) and (63), we have

$$
n_{2} \Delta(\ln f)+\frac{10}{9} n_{2} \cot ^{2} 9\|\nabla \ln f\|^{2}=\left(\frac{\kappa+3 \alpha}{4}\right) n_{2} n_{1}-\sum_{i=1}^{2 d_{1}} \sum_{j=1}^{2 d_{2}}\left\|h_{v}\left(e_{i}, e_{j}^{*}\right)\right\|^{2} .
$$

From (83) and (84), we find the following equation:

$\frac{10}{9} \cot ^{2} \vartheta\|\nabla \ln f\|^{2}=n_{1}-\frac{1}{n_{2}} \sum_{i=1}^{2 d_{1}} \sum_{j=1}^{2 d_{2}}\left\|h_{v}\left(e_{i}, e_{j}^{*}\right)\right\|^{2}+\int_{M} \mathscr{R} i c(\nabla \ln f, \cdot) \mathrm{dV}$.

Further simplifications give

$$
\begin{aligned}
\int_{M^{n}} \mathscr{R} i c(\nabla \ln f, \cdot) \mathrm{dV}+\left(\frac{\kappa+3 \alpha}{4}\right) n_{1}= & \frac{1}{n_{2}} \sum_{i=1}^{2 d_{1}} \sum_{j=1}^{2 d_{2}}\left\|h_{v}\left(e_{i}, e_{j}^{*}\right)\right\|^{2} \\
& +\frac{10}{9} \cot ^{2} \vartheta\|\nabla \ln f\|^{2}
\end{aligned}
$$

If the equality (80) is satisfied, then from (86), we obtain the following condition:

$$
\frac{10}{9} \cot ^{2} \vartheta\|\nabla \ln f\|^{2}=0
$$

Therefore, from the above equation, we derive two cases such that

$$
\cot ^{2} \vartheta=0 \text {, or }\|\nabla \ln f\|^{2}=0
$$

Case I: We consider $\cot ^{2} \vartheta=0\left(\cos ^{2} \vartheta / \sin ^{2} \vartheta\right)=0$, which implies that $\cos \vartheta=0 \vartheta=\pi / 2$. From Remark 8 , we conclude that $N_{\vartheta}^{n_{2}}$ becomes a totally real submanifold; hence, $M^{n}$ becomes a CR-warped product submanifold of a nearly 
Kaehler manifold. This completes the proof of (i) from Theorem 33.

Case II: We assume that $\|\nabla \ln f\|^{2}=0$, which means that $\nabla \ln f=0$ and $\operatorname{grad} \ln f=0$. This implies that $f$ is a constant function on $M^{n}$. Hence, from Remark 11, we conclude that $M^{n}$ is a trivial warped product semi-slant submanifold of a nearly Kaehler manifold. This is the second part (ii) of Theorem 33.

Theorem 34. Let $\varphi: M^{n}=N_{T}^{n_{1}} \times{ }_{f} N_{\vartheta}^{n_{2}}$ be an isometric immersion of a compact warped product semi-slant submanifold $N_{T}^{n_{1}} \times{ }_{f} N_{\vartheta}^{n_{2}}$ in a generalized complex space form $\tilde{M}^{2 m}(\kappa, \alpha)$ admitting a nearly Kaehler structure, such that the warping function $\ln f$ is the first eigenfunction of the Laplacian of $N_{T}^{n_{1}}$; this is associated with the first eigenvalue $\lambda_{1}$, which satisfies the following:

$$
\begin{aligned}
& \int \| \text { Hesslnf } \|^{2} d V+\int \operatorname{Ric}(\nabla \ln f, \nabla \ln f) d V \\
& =\frac{9 \lambda_{1} \tan ^{2} \vartheta}{10 n_{2}} \int\left(\left(\frac{\kappa+3 \alpha}{4}\right) n_{2} n_{1}-\sum_{i=1}^{n_{1}} \sum_{j=1}^{n_{2}}\left\|h_{v}\left(e_{i}, e_{j}^{*}\right)\right\|^{2}\right) d V .
\end{aligned}
$$

Proof. $\ln f$ is the first eigenfunction of the Laplacian of $M^{n}$ and is associated with the first eigenvalue $\lambda_{1}$; that is, $\Delta$ $\ln f=\lambda_{1} \ln f$. Thus, we recall the Bochner formula (see, e.g., [42]), which states that for a differentiable function $f$ defined on a Riemannian manifold, the following relation holds:

$$
\frac{1}{2} \Delta\|\nabla \ln f\|^{2}=\|\operatorname{Hess} \ln f\|^{2}+\operatorname{Ric}(\nabla \ln f, \nabla \ln f)+g(\nabla \ln f, \nabla(\Delta \ln f)) .
$$

Integrating the above equation with the help of the Stokes theorem, we obtain

$\int\|\operatorname{Hess} \ln f\|^{2} d V+\int \operatorname{Ric}(\nabla \ln f, \nabla \ln f) d V+\int g(\nabla \ln f, \nabla(\Delta \ln f)) d V=0$.

Now, by using $\Delta \ln f=\lambda_{1} \ln f$ and rearranging the above equation, we derive

$$
\int\|\nabla \ln f\|^{2} d V=-\frac{1}{\lambda_{1}}\left(\int\|\operatorname{Hess} \ln f\|^{2} d V+\int \operatorname{Ric}(\nabla \ln f, \nabla \ln f) d V\right) .
$$

Integrating equation (84), we obtain

$$
\frac{10 n_{2}}{9} \cot ^{2} \vartheta \int\|\nabla \ln f\|^{2} d V=\int\left(\left(\frac{\kappa+3 \alpha}{4}\right) n_{2} n_{1}-\sum_{i=1}^{2 d_{1}} \sum_{j=1}^{2 d_{2}}\left\|h_{v}\left(e_{i}, e_{j}^{*}\right)\right\|^{2}\right) d V .
$$

It follows from (92) and (93) that

$$
\begin{array}{r}
\frac{10 n_{2} \cot ^{2} \vartheta}{9 \lambda_{1}}\left(\int\|\operatorname{Hess} \ln f\|^{2} d V+\int \operatorname{Ric}(\nabla \ln f, \nabla \ln f) d V\right) \\
=\int\left(\left(\frac{\kappa+3 \alpha}{4}\right) n_{2} n_{1}-\sum_{i=1}^{2 d_{1}} \sum_{j=1}^{2 d_{2}}\left\|h_{v}\left(e_{i}, e_{j}^{*}\right)\right\|^{2}\right) d V .
\end{array}
$$

The above equation and (75) imply that $\cot ^{2} \vartheta=0$ $\left(\cos ^{2} \vartheta / \sin ^{2} \vartheta\right)=0$, which implies that $\cos \vartheta=0 \vartheta=\pi / 2$. Again, from Remark 8, we conclude that $N_{\vartheta}^{n_{2}}$ becomes a totally real submanifold; by using the statement of Theorem 34, we obtain our desired result.

Riemannian manifolds with no Ricci curvature are known as Ricci-flat manifolds. Ricci-flat manifolds are Einstein manifolds that do not require the cosmological constant to vanish. In a Ricci-flat manifold (particularly in Euclidean space), a circle, for example, can be deformed into an ellipse of equal area. We get the following result after taking into account the fact that warped product submanifolds are Ricci-flat.

Theorem 35. Let $\varphi: M^{n}=N_{T}^{n_{1}} \times{ }_{f} N_{\vartheta}^{n_{2}}$ be an isometric immersion of a compact warped product semi-slant submanifold $N_{T}^{n_{1}} \times{ }_{f} N_{\vartheta}^{n_{2}}$ in a generalized complex space form $\tilde{M}^{2 m}(\kappa, \alpha)$ admitting a nearly Kaehler structure, such that the warping function $\ln f$ is the first eigenfunction of the Laplacian of $N_{T}^{n_{1}}$ and is associated with the first eigenvalue $\lambda_{1}$; then, $N_{T}^{n_{1}}$ is Ricci-flat if and only if

$\int \|$ Hesslnf $\|^{2} d V=\frac{9 \lambda_{1} \tan ^{2} \vartheta}{10 n_{2}} \int\left(\left(\frac{\kappa+3 \alpha}{4}\right) n_{2} n_{1}-\sum_{i=1}^{2 d_{1}} \sum_{j=1}^{2 d_{2}}\left\|h_{v}\left(e_{i}, e_{j}^{*}\right)\right\|^{2}\right) d V$.

Proof. Thus, from (94) and (95), we obtain $\int \operatorname{Ric}(\nabla \ln f, \nabla$ $\ln f) d V=0$. This means that $N_{T}^{n_{1}}$ is Ricci-flat. The converse proof is straightforward. This completes the proof of the theorem.

\section{Data Availability}

There is no data used for this manuscript.

\section{Conflicts of Interest}

The authors declare no competing of interest.

\section{Authors' Contributions}

All authors contributed equally to this work. All authors finalized the manuscript. 


\section{Acknowledgments}

The authors extend their appreciation to the Deanship of Scientific Research at King Khalid University for funding this work through a research group program under grant number R.G.P.2/74/42. This work was also funded by the National Natural Science Foundation of China (Grant No. 12101168).

\section{References}

[1] B. Chen, "Geometry of warped product CR-submanifolds in Kaehler manifolds," Monatshefte für Mathematik, vol. 133, no. 3, pp. 177-195, 2001.

[2] P. Ehrlich, Y. T. Jung, S. B. Kim, and C. G. Shin, "Partial differential equations and scalar curvature of warped product manifolds," Nonlinear Analysis, vol. 44, no. 4, pp. 545-553, 2001.

[3] R. Bishop and B. O’Neill, "Manifolds of negative curvature," Transactions of the American Mathematical Society, vol. 145, pp. 1-9, 1969.

[4] B. Chen, "Slant immersions," Bulletin of the Australian Mathematical Society, vol. 41, no. 1, pp. 135-147, 1990.

[5] F. R. al-Solamy, V. Khan, and S. Uddin, "Geometry of warped product semi-slant submanifolds of nearly Kaehler manifolds," Results in Mathematics, vol. 71, no. 3-4, pp. 783-799, 2017.

[6] B. Chen, "Geometry of warped product submanifolds: a survey," Journal of Advanced Mathematical Studies, vol. 6, no. 2, pp. 1-43, 2013.

[7] B. Chen, "Another general inequality for warped product CRwarped product submanifold in complex space forms," Hokkaido Mathematical Journal, vol. 32, pp. 415-444, 2003.

[8] M. Atceken, "Contact CR-warped product submanifolds in cosymplectic space forms," Collectanea Mathematica, vol. 62, no. 1, pp. 17-26, 2011.

[9] I. Hasegawa and I. Mihai, "Contact CR-warped product submanifolds in Sasakian manifolds," Geometriae Dedicata, vol. 102, no. 1, pp. 143-150, 2003.

[10] A. Mihai, "Shape operator $A_{H}$ for slant submanifolds in generalized complex space forms," Turkish Journal of Mathematics, vol. 27, no. 4, pp. 509-524, 2004.

[11] I. Munteanu, "Warped product contact CR-submanifolds of Sasakian space forms," Universitatis Debreceniensis, vol. 66, pp. 75-120, 2005.

[12] A. Ali, P. Laurian-Ioan, and A. Alkhaldi, "Ricci curvature on warped product submanifolds in spheres with geometric applications," Journal of Geometry and Physics, vol. 146, article 103510, 2019.

[13] A. Ali, J. W. Lee, and A. H. Alkhaldi, "Geometric classification of warped product submanifolds of nearly Kaehler manifolds with a slant fiber," International Journal of Geometric Methods in Modern Physics., vol. 16, no. 2, article 1950031, 2019.

[14] R. Ali, F. Mofarreh, N. Alluhaibi, A. Ali, and I. Ahmad, "On differential equations characterizing Legendrian submanifolds of Sasakian space forms," Mathematics., vol. 8, no. 2, p. 150, 2020.

[15] A. Ali and P. Laurian-Ioan, "Geometry of warped product immersions of Kenmotsu space forms and its applications to slant immersions," Journal of Geometry and Physics, vol. 114, pp. 276-290, 2017.
[16] A. Ali and C. Ozel, "Geometry of warped product pointwise semi-slant submanifolds of cosymplectic manifolds and its applications," International Journal of Geometric Methods in Modern Physics, vol. 14, no. 3, article 1750042, 2017.

[17] I. al-Dayel and M. Ali Khan, "Ricci curvature of contact CRwarped product submanifolds in generalized Sasakian space forms admitting nearly Sasakian structure," AIMS Mathematics, vol. 6, no. 3, pp. 2132-2151, 2021.

[18] O. Calin and D. Chang, Geometric mechanics on Riemannian manifolds: applications to partial differential equations, Springer Science \& Business Media, 2006.

[19] D. Kim and Y. Kim, "Compact Einstein warped product spaces with nonpositive scalar curvature," Proceedings of the American Mathematical Society, vol. 131, no. 8, pp. 2573-2576, 2003.

[20] Y. Li, S. Liu, and Z. Wang, "Tangent developables and Darboux developables of framed curves," Topology and its Applications, vol. 301, article 107526, 2021.

[21] Y. Li and Z. Wang, "Lightlike tangent developables in de Sitter 3-space," Journal of Geometry and Physics, vol. 164, article 104188, 2021.

[22] Y. Li, Z. Wang, and T. Zhao, "Geometric Algebra of Singular Ruled Surfaces," Advances in Applied Clifford Algebras, vol. 31, no. 1, pp. 1-19, 2021.

[23] Y. Li, Y. Zhu, and Q. Sun, "Singularities and dualities of pedal curves in pseudo-hyperbolic and de sitter space," International Journal of Geometric Methods in Modern Physics, vol. 18, no. 1, article 2150008, 2021.

[24] Y. Li, Z. Wang, and T. Zhao, "Slant helix of order $\mathrm{n}$ and sequence of Darboux developables of principal-directional curves," Mathematicsl Methods in the Applied Sciences, vol. 43, no. 17, pp. 9888-9903, 2020.

[25] B. Sahin, "Non-existence of warped product semi-slant submanifolds of Kaehler manifolds," Geometriae Dedicata, vol. 117, no. 1, pp. 195-202, 2006.

[26] B. Sahin and R. Gunes, "CR-warped product submanifolds of nearly Kaehler manifolds," Beiträge zur Algebra und Geometrie, vol. 49, pp. 383-397, 2008.

[27] F. Urbano, CR-submanifolds of nearly Kaehler manifolds, [PhD Thesis], Granada, 1980.

[28] I. Mihai, "Ideal Kaehlerian slant submanifolds in complex space forms," The Rocky Mountain Journal of Mathematics, vol. 35, pp. 941-952, 2005.

[29] A. Mihai and B. Y. Chen, "Inequalities for slant submanifolds in generalized complex space forms," Radovi Mathematicki, vol. 2, pp. 215-231, 2004.

[30] A. Mihai, "Warped product submanifolds in generalized complex space forms," Acta Mathematica Academiae Paedagogicae Ny'ıregyh'aziensis, vol. 21, pp. 79-87, 2005.

[31] A. Mustafa, S. Uddin, and R. Wong, "Generalized inequalities on warped product submanifolds in nearly trans-Sasakian manifolds," Journal of Inequalities and Applications, vol. 2014, no. 1, Article ID 346, p. 16, 2014.

[32] I. Mihai, "Contact CR-warped product submanifolds in Sasakian space forms," Geometriae Dedicata, vol. 109, no. 1, pp. 165-173, 2004.

[33] K. Yano and M. Kon, Structures on Manifolds, World Scientific, 1985.

[34] N. Papaghiuc, "Semi-slant submanifold of Kaehlerian manifold," Annals of the "Alexandru Ioan Cuza" University of Iaşi, vol. 40, pp. 55-61, 1994. 
[35] Y. Li, M. Lone, and U. Wani, "Biharmonic submanifolds of Kaehler product manifolds," AIMS Mathematics, vol. 6, pp. 309-9321, 2021.

[36] Y. Li, A. Ali, and R. Ali, "A general inequality for CR-warped products in generalized Sasakian space form and its applications," Advances in Mathematical Physics, vol. 2021, Article ID 5777554, 6 pages, 2021.

[37] Y. Li, P. Laurian-Ioan, A. Ali, and A. Alkhaldi, "Null homology groups and stable currents in warped product submanifolds of Euclidean spaces," Symmetry, vol. 13, no. 9, p. 1587, 2021.

[38] Y. Li, A. Ali, F. Mofarreh, and N. Alluhaibi, "Homology groups in warped product submanifolds in hyperbolic spaces," Journal of Mathematics, vol. 2021, Article ID 8554738, 10 pages, 2021.

[39] A. Ali, S. Uddin, and W. Othman, "Geometry of warped product pointwise semi-slant submanifolds of Kaehler manifolds," Univerzitet $u$ Nišu, vol. 31, no. 12, pp. 3771-3788, 2017.

[40] N. S. al-Luhaibi, F. R. al-Solamy, and V. Khan, "CR-warped product submanifolds of nearly Kaehler manifolds," Journal of the Korean Mathematical Society, vol. 46, no. 5, pp. 979995, 2009.

[41] V. Khan and K. Khan, "Generic warped product submanifolds in nearly Kaehler manifolds," Contributions to Algebra and Geometry, vol. 50-2, pp. 337-352, 2009.

[42] M. Berger, "Les variétés riemanniennes (1/4)-pincées," Annali della Scuola Normale Superiore di Pisa-Classe di Scienze, vol. 14, no. 4, pp. 161-170, 1960. 